\title{
Strategic dialogic communication through digital media during COVID-19 crisis
}

\author{
By Mark Anthony Camilleri ${ }^{1}$ \\ https://orcid.org/0000-0003-1288-4256
}

This is a pre-publication version.

How to Cite: Camilleri, M.A. (2020). Strategic dialogic communication through digital media during COVID-19 crisis. In Camilleri, M.A. (Ed.) Strategic Corporate Communication in the Digital Age, Emerald, Bingley, UK.

\begin{abstract}
Institutions and organizations are increasingly using the digital media to communicate with stakeholders on a day-to-day basis and during crises situations. Therefore, this chapter presents a bibliographic analysis on digital corporate communication technologies. The grounded theory's inductive approach was used to capture and interpret the findings from Scopusindexed publications. The articles were scrutinized in their entirety, including their research questions, methodologies and interpretation of the findings. Afterwards, this contribution identifies the opportunities and challenges that emerged during an unprecedented Coronavirus (COVID-19) outbreak. In conclusion, it implies that there is scope for institutions and organizations to incorporate digital and social media in their crises' communications and risk management plans. This will enable them to be in a better position to engage in credible and transparent dialogic communications with different stakeholders.
\end{abstract}

Keywords: Corporate communication, digital media, digital communication, social media, crises, COVID-19.

\footnotetext{
${ }^{1}$ Department of Corporate Communication, Faculty of Media and Knowledge Sciences, University of Malta, MALTA. Email: mark.a.camilleri@um.edu.mt AND
}

The Business School, University of Edinburgh, SCOTLAND. 


\subsection{Introduction}

Corporate communications practitioners can avail themselves of a wide range of digital media, to convey commercial information and/or to interact with stakeholders and the general public. They can use them to create electronic content to inform and educate online users about their products or services (Köhler \& Zerfass, 2019; García García, Carrillo-Durán \& Tato Jimenez, 2017; Krishna \& Vibber, 2017; Cornellisen, 2008). Alternatively, they may utilise the digital platforms to communicate about their organizations' activities, including corporate social responsibility practices and/or to engage with online users, in real time (Camilleri, 2018a; 2020). Institutions and organizations may usually promote their activities and/or offerings through websites or other digital media including blogs, vlogs, video clips and social media, among others (Killian \& McManus, 2015; Ruehl \& Ingenhoff, 2015; Fraustino \& ConnollyAhern, 2015). Their websites can have responsive designs and different formats of verbal, vocal and visual content to appeal to their targeted audiences. Their corporate communications content can be displayed in web pages; blog posts, social media posts, eBooks, online articles, review sites, product FAQs, videos and micro-videos; pictures, infographics, and animated GIFs among other media. Marketers are also expected to create appropriate content and to ensure that their online sites are presented in an attractive and user-friendly format and structure. They can optimize it for mobile screens, as this medium has surpassed desktop traffic. Moreover, their online domains have to be responsive in terms of load time, content length, voice search, image and video processing.

Institutions and organizations can develop corporate websites or blogs that may be accessed through organic queries via search engines like Google and Bing, among others (Romenti, Valentini, Murtarelli \& Meggiorin, 2016). These search engines will usually identify quality content in their search results. Hence, corporate communications practitioners and digital marketers ought to create fresh, engaging content with a growing number of quality links to 
enhance the quality of their websites. They have to make sure that their landing (home) page features a great design to improve the online users' experience (Camilleri, 2019a). Moreover, they may utilize social media networks like Facebook, Twitter, Instagram and Linkedin, among others, to disseminate their content to their subscribers and to engage in interactive conversations with them (Camilleri \& Isaias, 2020; García-Orosa, 2019; Champoux, Durgee \& McGlynn, 2012). Corporate communication practitioners can also work with online influencers who are capable of attracting large audiences. This latest development reaffirms the link between high quality, corporate communication and the digital media (Camilleri, 2017a). Organizations including corporations as well as small businesses ought to be familiar with the digital and mobile technologies (Melewar \& Navalekar, 2002). They can use them to on a day-to day basis to forge relationships with different stakeholders including employees, customers, suppliers, investors, media, regulatory authorities and the community at large (Bachmann, 2019; Costa-Sánchez \& Míguez-González, 2018; Loureiro \& Gomes, 2016), or during crises situations (Krishna \& Vibber, 2017; Champoux et al., 2012).

In this light, this chapter appraises previous theoretical underpinnings that were focused on strategic dialogic corporate communications in the digital age. Afterwards, it synthesizes the findings from relevant academic literature, and discusses about the latest opportunities and challenges facing organizations, following the outbreak of Coronavirus (COVID-19) pandemic. In conclusion, this timely contribution puts forward key implications to practitioners and identifies future research avenues.

\subsection{Corporate communication through digital media}

The disruptive technologies are supporting institutions as well as organizations in their corporate communications. They allow them to improve their interactive engagement with 
stakeholders, whilst enhancing their legitimacy in society (Mohd-Sulaiman \& Hingun, 2020; See, Sunar, Kusnayat, Aziz, 2018; Hoffmann \& Aeschlimann, 2017). The latest digital communications are synchronous and dynamic as they enable online users to exchange information in real time (Romenti et al., 2016; Turner, Wilkie \& Rosen, 2004). They have facilitated the corporate communications practitioners' content marketing and increased their two-way interactions with different audiences (Abratt \& Cullinan, 2017; Järvinen \& Taiminen, 2016; Holliman \& Rowley, 2014; Rowley, 2008).

\subsubsection{Social Media}

Social media platforms enable symmetric, dialogic communications in an online environment where there is limited gatekeeping (Camilleri \& Costa, 2018; Overton-de Klerk \& Verwey, 2013). Individuals and organizations can use them to establish their authority and trust among stakeholders by consistently creating high quality content that is relevant to them. They are encouraged to create engaging content to communicate with targeted audiences. Their online content can turn "viral" as online users may be intrigued to re-post it again through the social media. Such electronic word-of-mouth publicity and user generated content are usually perceived as highly trustworthy sources for prospective customers (Manfredi-Sánchez, 2019; Chu \& Kim, 2011; Ye, Law, Gu \& Chen, 2011). Thus, corporate communications practitioners are increasingly subscribing to different social media networks, including Facebook, YouTube, Instagram, Twitter and LinkedIn, among others, to increase the reach of their content (NavarroBeltrá, Medina, Correia, 2020; Costa-Sánchez, amd Míguez-González, 2018; Champoux et al., 2012).

Currently, Facebook has 2.45 billion users. Other popular social media networks include Instagram (1 billion users), Reddit (430 million users), Snapchat (360 million users), Twitter (330 million users), Pinterest (322 million users) and LinkedIn (310 million users) (SEJ, 2020). 
These networks have become very popular communication outlets as they promote online content and allow synchronous interactions, in real time. In addition, some of them, including Facebook, provide messenger systems, including Facebook Messenger or WhatsApp. They also offer live video functions to enhance virtual communications.

Twitter is a platform that is based on topical content. Generally, its users are encouraged to use keywords and hashtags on certain topics, in particular locations. Twitter posts have a 280character limit. Therefore, its subscribers have to articulate short, focused messages (Siano, Vollero, Della Volpe, Confetto, Foroudi \& Palazzo, 2018). Its subscribers are expected to dedicate time to look after their account as they need to respond to their followers to avoid negative criticism (Camilleri, 2018b). Like Facebook, Twitter enables direct, two-way communications among subscribers (Caerols-Mateo, Viñarás-Abad \& Gonzálvez-Valles, 2017). Hence, it can be used to engage in interactive conversations with other users.

LinkedIn is another effective tool, particularly for personal branding. This social network helps its users to identify and engage with influencers who share similar interests. Companies and individuals can also use this site to create online articles like a blog. Pinterest and Instagram enable their users to share images and ideas with others in their networks. They are focused on the dissemination of visual content. Instagram and Snapchat can feature videos and usergenerated content. They may include influencer marketing material (Rios Marques, Casais \& Camilleri, 2020; Hajarian, Camilleri, Diaz \& Aedo, 2020). Moreover, practitioners are increasingly uploading short, fun videos which often turn viral on YouTube. This site offers an excellent way to humanise or animate corporate communications content through video content.

The usage of social media has radically influenced the style of personal and corporate communications as well as the dissemination of knowledge and information. Platforms can be 
personalized, self-managed and interconnected. They can blend written content with images, videos and hyperlinks (Brennan \& Merkl-Davies, 2018). This disruptive innovation has led individuals from different demographic segments in society to refine their digital and communication skills, as social media has impacted their way of thinking, talking and even their social lives (Dyuzhev \& Boichenko, 2019).

In a similar vein, social media platforms may be used by businesses and other organizations to illustrate their communication to stakeholders through verbal, visual and vocal content. For example, non-governmental organizations can raise awareness about political, social and environmental issues. Businesses can use social media to communicate about their corporate social (and environmental) responsibility (CSR), corporate governance, responsible procurement, philanthropic and stewardship practices, et cetera, in different markets (Troise \& Camilleri, 2020; Weder, Einwiller \& Eberwein, 2019; Camilleri, 2019b, 2017b, 2016a; 2015; Mendes-da-Silva, Christensen \& Richardson, 2008; Fombrun, 2005). These online networks are also effective monitoring tools. They feature the most trending topics and contemporary issues (Lestari, Suryana, Mulyana \& Hidayat, 2019). Social media users can utilize the hashtag (\#) to enhance the visibility when they share content. For example, some of the most popular hashtags for corporate communication practitioners include \#communication, \#marketing, \#digital, \#CSR, \#sustainable, \#EcoFriendly, \#sustainability, \#ZeroWaste \#CircularEconomy, \#CSRcomm, et cetera. Hashtags may be used to raise awareness on charities, philanthropic institutions as well as on non-governmental organizations and trusts (Camilleri, 2016b, 2016c).

The social media have transformed the communicative dynamics within and between corporations and their external environment (Capriotti, Zeler \& Camilleri, 2020; Bruce \& Solomon, 2013). These platforms have potential to empower their subscribers to engage with 
others, on a wide array of topics. Individuals, groups, organizations and institutions can use them to promote their content online and through ubiquitous mobile technologies.

\subsubsection{Mobile}

The mobile devices have become a part of our daily lives. We use them while we are out and about (Butler, Camilleri, Creed \& Zutshi, 2020). Individuals can access the Internet through their laptop, smart phone or tablet from different places. They can read their emails and posts on social media networks. Alternatively, they can share pictures, listen to podcasts and watch videos or live streams. Therefore, organizations and their content marketers are encouraged to create responsive and scalable mobile-friendly applications (apps) to improve their users' browsing experience through different technologies and applications (apps). For example, search engines like Google and Apple's Safari are two of the most popular apps that are found in mobile devices. Their search engines serve their online or mobile users in their search queries, as they list and rank websites for them.

\subsubsection{Search Engines}

The search engines' algorithms identify pertinent information from corporate websites and present snippets and links to them, in their search results. The items that appear in the first page of the search results are placed in a better position than others which are featured in the latter pages of the same search query.

The search engine optimization term (or simply SEO) is the process of getting traffic from "free" "organic", editorial" or "natural" listings on search engines. The search engines are supposed to feature and signal the most relevant and highest quality content to their users. As a result, top-ranking search results tend to have lots of social shares, while those ranked lower have fewer. Several organizations are striving in their endeavors to improve their placement in search engine results. 
There are opportunities for them to leverage their content through search results. They may create high quality, online content that can be captured through search engines, including blogs, if they use appropriate keywords and present relevant material.

\subsubsection{Blogging}

Blogging, guest blogging and the curation of websites are a great way to disseminate corporate communications. These digital media outlets may be used to raise awareness about social and environmental issues. The regular contributions on blogs allow their users to connect with other individuals and organizations who share similar values, ideas and opinions (Brennan \& MerklDavies, 2018; Kristina \& Payal, 2013). Notwithstanding, blogs and websites possess analytical tools that can reveal which content had the biggest impact on their audiences.

\subsection{Data capture and analysis}

This research relied on the grounded theory's methodological approach to capture and interpret the findings (Eisenhardt, Graebner \& Sonenshein, 2016). An inductive approach was used to collect the data from Scopus-indexed publications. The search results from the systematic review was focused on those publications that featured the words 'corporate communication' and 'digital' in article titles, abstracts and keywords. The researcher scrutinized the journal articles' content in their entirety, including their research questions, methodologies and interpretation of the findings.

There were 91 contributions that were listed in Scopus' repository. These articles were published between January 2000 to June 2020. Thirty-nine (39) of them were journal articles that were in English. Their top 3 subject areas were related to: business, management and accounting (27), social sciences (22) and computer science (3). Table 1.1 presents a complete list of these contributions. It endorses their authors, describes their research approaches, and 
features the keywords of their articles. This research has excluded 2 out of 39 as they did not have identifiable authors.

For the record, there were just 8 journal articles that were captured when the search query included "corporate communication" and "digital media". Moreover, there was only one article that included "corporate communication" and "COVID-19" in the search results (i.e. Xifra, 2020).

Table 1.1: List of Scopus-indexed publications on digital corporate communication

\begin{tabular}{|l|l|l|l|l|}
\hline Authors & Year & Source title & $\begin{array}{l}\text { Research } \\
\text { approach }\end{array}$ & Keywords \\
\hline $\begin{array}{l}\text { Segars, A.H., } \\
\text { Kohut, G.F. }\end{array}$ & 2001 & $\begin{array}{l}\text { Journal of } \\
\text { Management } \\
\text { Studies }\end{array}$ & $\begin{array}{l}\text { Empirical } \\
\text { (quantitative) }\end{array}$ & $\begin{array}{l}\text { shareholders, strategic } \\
\text { communication, } \\
\text { credibility, efficacy, } \\
\text { commitment, } \\
\text { responsibility. }\end{array}$ \\
\hline $\begin{array}{l}\text { Champoux, V., } \\
\text { Durgee, J., } \\
\text { McGlynn, L. }\end{array}$ & 2012 & $\begin{array}{l}\text { Journal of } \\
\text { Business Strategy }\end{array}$ & $\begin{array}{l}\text { Review } \\
\text { (case study) }\end{array}$ & $\begin{array}{l}\text { Facebook, social media, } \\
\text { crisis management, } \\
\text { corporate } \\
\text { communications, } \\
\text { consumer complaints. }\end{array}$ \\
\hline $\begin{array}{l}\text { Killian, G., } \\
\text { McManus, K. }\end{array}$ & 2015 & $\begin{array}{l}\text { Business } \\
\text { Horizons }\end{array}$ & $\begin{array}{l}\text { Empirical } \\
\text { (in-depth } \\
\text { interviews) }\end{array}$ & $\begin{array}{l}\text { social media strategy, } \\
\text { customer relationship } \\
\text { management, corporate } \\
\text { communications, } \\
\text { integrated marketing } \\
\text { communications. }\end{array}$ \\
\hline $\begin{array}{l}\text { Jones, S.L. } \\
\text { Ingenhoff, D. }\end{array}$ & 2005 & $\begin{array}{l}\text { Journal of } \\
\text { Business and } \\
\text { Technical } \\
\text { Communication }\end{array}$ & $\begin{array}{l}\text { Review } \\
\text { (conceptual) }\end{array}$ & $\begin{array}{l}\text { collaboration, collaborati } \\
\text { ve writing, professional } \\
\text { writers, intranet, XML. }\end{array}$ \\
\hline $\begin{array}{l}\text { Marketing Theory } \\
\text { and Practice } \\
\text { Solomon, M. }\end{array}$ & 2015 & $\begin{array}{l}\text { Journal of } \\
\text { Communication } \\
\text { Management } \\
\text { (conceptual) }\end{array}$ & $\begin{array}{l}\text { Empirical } \\
\text { (semi- } \\
\text { structured } \\
\text { interviews) }\end{array}$ & $\begin{array}{l}\text { corporate } \\
\text { communications, digital } \\
\text { real estate, media } \\
\text { anarchy, network effects, } \\
\text { scalability, reputation } \\
\text { economy. }\end{array}$ \\
$\begin{array}{l}\text { social networking sites, } \\
\text { communication } \\
\text { management, stakeholder } \\
\text { analysis, social media, } \\
\text { public relations, }\end{array}$ \\
\hline
\end{tabular}




\begin{tabular}{|c|c|c|c|c|}
\hline & & & & $\begin{array}{l}\text { corporate } \\
\text { communications. }\end{array}$ \\
\hline $\begin{array}{l}\text { Melewar, T.C., } \\
\text { Navalekar, A. }\end{array}$ & 2002 & $\begin{array}{l}\text { Marketing } \\
\text { Intelligence and } \\
\text { Planning }\end{array}$ & $\begin{array}{l}\text { Review } \\
\text { (discursive) }\end{array}$ & $\begin{array}{l}\text { corporate identity, } \\
\text { financial services, } \\
\text { corporate } \\
\text { communications, } \\
\text { ecommerce. }\end{array}$ \\
\hline $\begin{array}{l}\text { Ngai, C.S.-B., } \\
\text { Singh, R.G. }\end{array}$ & 2014 & $\begin{array}{l}\text { Journal of } \\
\text { Business and } \\
\text { Technical } \\
\text { Communication }\end{array}$ & $\begin{array}{l}\text { Review } \\
\text { (content } \\
\text { analysis) }\end{array}$ & $\begin{array}{l}\text { CEO messages, corporate } \\
\text { communication, Greater } \\
\text { China, bilingual text, } \\
\text { content analysis. }\end{array}$ \\
\hline $\begin{array}{l}\text { Overton-de } \\
\text { Klerk, N., } \\
\text { Verwey, S. }\end{array}$ & 2013 & Communicatio & $\begin{array}{l}\text { Review } \\
\text { (theoretical) }\end{array}$ & $\begin{array}{l}\text { convergence, dialogue, } \\
\text { dissent, organising, } \\
\text { paradigm, paradigm } \\
\text { shifts, paradigmatic } \\
\text { debates, post-modern } \\
\text { communication practice, } \\
\text { stakeholder } \\
\text { empowerment, strategic } \\
\text { communication. }\end{array}$ \\
\hline $\begin{array}{l}\text { Fraustino, J.D., } \\
\text { Connolly- } \\
\text { Ahern, C. }\end{array}$ & 2015 & $\begin{array}{l}\text { Journal of Public } \\
\text { Relations } \\
\text { Research }\end{array}$ & $\begin{array}{l}\text { Empirical } \\
\text { (quantitative) }\end{array}$ & $\begin{array}{l}\text { social media, corporate } \\
\text { messages, Facebook, } \\
\text { online communication, } \\
\text { corporate ability, } \\
\text { corporate social } \\
\text { responsibility. }\end{array}$ \\
\hline $\begin{array}{l}\text { Romenti, S., } \\
\text { Valentini, C., } \\
\text { Murtarelli, G., } \\
\text { Meggiorin, K. }\end{array}$ & 2016 & $\begin{array}{l}\text { Journal of } \\
\text { Communication } \\
\text { Management }\end{array}$ & $\begin{array}{l}\text { Empirical } \\
\text { (quantitative) }\end{array}$ & $\begin{array}{l}\text { social media, } \\
\text { measurement, scale } \\
\text { development, dialogic } \\
\text { conversations. }\end{array}$ \\
\hline $\begin{array}{l}\text { Brennan, N.M., } \\
\text { Merkl-Davies, } \\
\text { D.M. }\end{array}$ & 2018 & $\begin{array}{l}\text { Accounting and } \\
\text { Business } \\
\text { Research }\end{array}$ & $\begin{array}{l}\text { Review } \\
\text { (conceptual) }\end{array}$ & $\begin{array}{l}\text { corporate } \\
\text { reporting, accounting } \\
\text { communication, connecti } \\
\text { vity. }\end{array}$ \\
\hline $\begin{array}{l}\text { Grafström, M., } \\
\text { Falkman, L.L. }\end{array}$ & 2017 & $\begin{array}{l}\text { Journal of } \\
\text { Organizational } \\
\text { Change } \\
\text { Management }\end{array}$ & $\begin{array}{l}\text { Review } \\
\text { (content } \\
\text { analysis) }\end{array}$ & $\begin{array}{l}\text { leadership, Twitter, } \\
\text { rhetoric, CEO } \\
\text { communication, } \\
\text { corporate narratives. }\end{array}$ \\
\hline $\begin{array}{l}\text { García García, } \\
\text { M., Carrillo- } \\
\text { Durán, M.V., } \\
\text { Tato Jimenez, } \\
\text { J.L. }\end{array}$ & 2017 & $\begin{array}{l}\text { Journal of } \\
\text { Communication } \\
\text { Management }\end{array}$ & $\begin{array}{l}\text { Empirical } \\
\text { (quantitative) }\end{array}$ & $\begin{array}{l}\text { digital media, strategic } \\
\text { communication, } \\
\text { corporate } \\
\text { communication, } \\
\text { communication } \\
\text { management. }\end{array}$ \\
\hline $\begin{array}{l}\text { Loureiro, } \\
\text { S.M.C., } \\
\text { Gomes, D.G. }\end{array}$ & 2016 & $\begin{array}{l}\text { Journal of } \\
\text { Promotion } \\
\text { Management }\end{array}$ & $\begin{array}{l}\text { Empirical } \\
\text { (quantitative) }\end{array}$ & $\begin{array}{l}\text { corporate communication } \\
\text { management, digital } \\
\text { strategies of } \\
\text { communication, public } \\
\text { relations, social network } \\
\text { sites. }\end{array}$ \\
\hline
\end{tabular}




\begin{tabular}{|c|c|c|c|c|}
\hline $\begin{array}{l}\text { Turner, A., } \\
\text { Wilkie, F., } \\
\text { Rosen, N. }\end{array}$ & 2004 & $\begin{array}{l}\text { New Library } \\
\text { World }\end{array}$ & $\begin{array}{l}\text { Review } \\
\text { (descriptive) }\end{array}$ & $\begin{array}{l}\text { libraries, corporate } \\
\text { communications, } \\
\text { promotional methods, } \\
\text { partnership. }\end{array}$ \\
\hline $\begin{array}{l}\text { Caerols-Mateo, } \\
\text { R., Viñarás- } \\
\text { Abad, M., } \\
\text { Gonzálvez- } \\
\text { Valles, J.E. }\end{array}$ & 2017 & $\begin{array}{l}\text { Revista Latina de } \\
\text { Comunicacion } \\
\text { Social }\end{array}$ & $\begin{array}{l}\text { Review } \\
\text { (content } \\
\text { analysis) }\end{array}$ & $\begin{array}{l}\text { museums, Twitter, } \\
\text { participation, content, } \\
\text { communication. }\end{array}$ \\
\hline $\begin{array}{l}\text { Caron, A.H., } \\
\text { Hwang, J.M., } \\
\text { Brummans, } \\
\text { B.H.J.M., } \\
\text { Caronia, L. }\end{array}$ & 2013 & $\begin{array}{l}\text { Corporate } \\
\text { Communications: } \\
\text { An International } \\
\text { Journal }\end{array}$ & $\begin{array}{l}\text { Empirical } \\
\text { (semi- } \\
\text { structured } \\
\text { interviews) }\end{array}$ & $\begin{array}{l}\text { senior management, } \\
\text { electronic mail, corporate } \\
\text { communications, written } \\
\text { communications, } \\
\text { business e-mail, mobile } \\
\text { communications, digital } \\
\text { technology, business } \\
\text { executives, self- } \\
\text { presentation, impression } \\
\text { management, paratext }\end{array}$ \\
\hline $\begin{array}{l}\text { Costa-Sánchez, } \\
\text { C., Míguez- } \\
\text { González, M.-I. }\end{array}$ & 2018 & $\begin{array}{l}\text { Profesional de la } \\
\text { Informacion }\end{array}$ & $\begin{array}{l}\text { Empirical } \\
\text { (content } \\
\text { analysis and } \\
\text { in-depth } \\
\text { interviews) }\end{array}$ & $\begin{array}{l}\text { communication } \\
\text { strategies, public } \\
\text { relations, health } \\
\text { communication, } \\
\text { corporate } \\
\text { communication, social } \\
\text { media. }\end{array}$ \\
\hline $\begin{array}{l}\text { Hoffmann, } \\
\text { C.P., } \\
\text { Aeschlimann, } \\
\text { L. }\end{array}$ & 2017 & $\begin{array}{l}\text { Corporate } \\
\text { Communications: } \\
\text { An International } \\
\text { Journal }\end{array}$ & $\begin{array}{l}\text { Empirical } \\
\text { (quantitative) }\end{array}$ & $\begin{array}{l}\text { investor relations, } \\
\text { engagement, social } \\
\text { media, online } \\
\text { communication. }\end{array}$ \\
\hline $\begin{array}{l}\text { Poloski Vokic, } \\
\text { N., Vidovic, M. }\end{array}$ & 2015 & $\begin{array}{l}\text { Public Relations } \\
\text { Review }\end{array}$ & $\begin{array}{l}\text { Empirical } \\
\text { (quantitative) }\end{array}$ & $\begin{array}{l}\text { digital publics, Xers, } \\
\text { digital, human resource } \\
\text { management (HRM) } \\
\text { practices, internal } \\
\text { communication (IC) } \\
\text { practices. }\end{array}$ \\
\hline $\begin{array}{l}\text { Kristina, S., } \\
\text { Payal, A. }\end{array}$ & 2013 & First Monday & $\begin{array}{l}\text { Review } \\
\text { (content } \\
\text { analysis) }\end{array}$ & $\begin{array}{l}\text { blogs, fashion, experts, } \\
\text { culture, business, } \\
\text { marketing. }\end{array}$ \\
\hline Colbran, M.P. & 2020 & $\begin{array}{l}\text { Policing and } \\
\text { Society }\end{array}$ & $\begin{array}{l}\text { Empirical } \\
\text { (interviews) }\end{array}$ & $\begin{array}{l}\text { police and social } \\
\text { media, corporate } \\
\text { communications, crime } \\
\text { reporting, police and } \\
\text { media, Leveson. }\end{array}$ \\
\hline $\begin{array}{l}\text { Weder, F., } \\
\text { Einwiller, S., } \\
\text { Eberwein, T. }\end{array}$ & 2019 & $\begin{array}{l}\text { Corporate } \\
\text { Communications: } \\
\text { An International } \\
\text { Journal }\end{array}$ & $\begin{array}{l}\text { Review } \\
\text { (theoretical) }\end{array}$ & $\begin{array}{l}\text { CSR communication, } \\
\text { reporting, impact, } \\
\text { communicative } \\
\text { responsibility, internal } \\
\text { CSR. }\end{array}$ \\
\hline
\end{tabular}




\begin{tabular}{|c|c|c|c|c|}
\hline $\begin{array}{l}\text { See, Z.S., } \\
\text { Sunar, M.S., } \\
\text { Kusnayat, A., } \\
\text { Aziz, K.A. }\end{array}$ & 2018 & $\begin{array}{l}\text { International } \\
\text { Journal of } \\
\text { Integrated } \\
\text { Engineering }\end{array}$ & $\begin{array}{l}\text { Empirical } \\
\text { (quantitative) }\end{array}$ & $\begin{array}{l}\text { corporate } \\
\text { communications, } \\
\text { interactive panorama, } \\
\text { spherical panorama, } \\
\text { virtual reality } 360 .\end{array}$ \\
\hline $\begin{array}{l}\text { Krishna, A., } \\
\text { Vibber, K.S. }\end{array}$ & 2017 & $\begin{array}{l}\text { Journal of } \\
\text { Communication } \\
\text { Management }\end{array}$ & $\begin{array}{l}\text { Mixed } \\
\text { methods } \\
\text { (descriptive } \\
\text { quantitative } \\
\text { techniques } \\
\text { and } \\
\text { qualitative } \\
\text { thematic } \\
\text { analysis) }\end{array}$ & $\begin{array}{l}\text { digital media, corporate } \\
\text { communication, social } \\
\text { media, methodology, } \\
\text { crisis communication, } \\
\text { public opinion, electronic } \\
\text { media. }\end{array}$ \\
\hline $\begin{array}{l}\text { Köhler, K., } \\
\text { Zerfass, A. }\end{array}$ & 2019 & $\begin{array}{l}\text { Journal of } \\
\text { Communication } \\
\text { Management }\end{array}$ & $\begin{array}{l}\text { Review } \\
\text { (content } \\
\text { analysis) }\end{array}$ & $\begin{array}{l}\text { best practice, digital } \\
\text { media, strategy, } \\
\text { corporate strategy, } \\
\text { corporate } \\
\text { communication, strategic } \\
\text { communication. }\end{array}$ \\
\hline Bachmann, $\mathrm{P}$. & 2019 & $\begin{array}{l}\text { Public Relations } \\
\text { Inquiry }\end{array}$ & $\begin{array}{l}\text { Review } \\
\text { (discursive) }\end{array}$ & $\begin{array}{l}\text { automation, big } \\
\text { data, liquid } \\
\text { modernity, postmodern } \\
\text { ethics, public } \\
\text { relations, Zygmunt } \\
\text { Bauman } \\
\end{array}$ \\
\hline $\begin{array}{l}\text { García-Orosa, } \\
\text { B. }\end{array}$ & 2019 & $\begin{array}{l}\text { Profesional de la } \\
\text { Informacion }\end{array}$ & $\begin{array}{l}\text { Review } \\
\text { (content } \\
\text { analysis) }\end{array}$ & $\begin{array}{l}\text { organizational } \\
\text { communication, } \\
\text { corporate } \\
\text { communication, digital } \\
\text { communication, } \\
\text { academic research, } \\
\text { research methods, } \\
\text { literature review, social } \\
\text { networks, big data, } \\
\text { review article. }\end{array}$ \\
\hline $\begin{array}{l}\text { Manfredi- } \\
\text { Sánchez, J.L. }\end{array}$ & 2019 & $\begin{array}{l}\text { Communication } \\
\text { and Society }\end{array}$ & $\begin{array}{l}\text { Review } \\
\text { (content } \\
\text { analysis) }\end{array}$ & $\begin{array}{l}\text { brand activism, corporate } \\
\text { political shift, corporate } \\
\text { diplomacy, campaign, } \\
\text { environment, consumer- } \\
\text { citizen, identity, } \\
\text { authenticity. }\end{array}$ \\
\hline $\begin{array}{l}\text { Mendes-da- } \\
\text { Silva, W., } \\
\text { Christensen, } \\
\text { T.E., } \\
\text { Richardson, } \\
\text { V.J. }\end{array}$ & 2008 & $\begin{array}{l}\text { Corporate } \\
\text { Ownership and } \\
\text { Control }\end{array}$ & $\begin{array}{l}\text { Empirical } \\
\text { (quantitative) }\end{array}$ & $\begin{array}{l}\text { voluntary disclosure, } \\
\text { Internet disclosure, } \\
\text { corporate websites, } \\
\text { corporate governance. }\end{array}$ \\
\hline
\end{tabular}




\begin{tabular}{|c|c|c|c|c|}
\hline $\begin{array}{l}\text { Mohd- } \\
\text { Sulaiman, } \\
\text { A.N., Hingun, } \\
\text { M. }\end{array}$ & 2020 & $\begin{array}{l}\text { International } \\
\text { Journal of Law } \\
\text { and Management }\end{array}$ & $\begin{array}{l}\text { Review (case } \\
\text { study) }\end{array}$ & $\begin{array}{l}\text { shareholders } \\
\text { engagement, corporate } \\
\text { communication, social } \\
\text { media, directors' } \\
\text { accountability. }\end{array}$ \\
\hline $\begin{array}{l}\text { Navarro-Beltrá, } \\
\text { M., Medina, } \\
\text { I.G., Correia, } \\
\text { P.A.P. }\end{array}$ & 2020 & $\begin{array}{l}\text { International } \\
\text { Journal of } \\
\text { Interactive } \\
\text { Mobile } \\
\text { Technologies }\end{array}$ & $\begin{array}{l}\text { Review } \\
\text { (content } \\
\text { analysis) }\end{array}$ & $\begin{array}{l}\text { fashion, Facebook, social } \\
\text { networks, social media, } \\
\text { Internet, corporate } \\
\text { communication, } \\
\text { dialogue, stakeholders, } \\
\text { content analysis. }\end{array}$ \\
\hline $\begin{array}{l}\text { Dyuzhev, V.G., } \\
\text { Boichenko, O.I. }\end{array}$ & 2019 & $\begin{array}{l}\text { Science and } \\
\text { Innovation }\end{array}$ & $\begin{array}{l}\text { Review (case } \\
\text { study) }\end{array}$ & $\begin{array}{l}\text { Bitrix 24, corporate } \\
\text { social } \\
\text { networks, innovative } \\
\text { receptivity. }\end{array}$ \\
\hline $\begin{array}{l}\text { Lestari, M.T., } \\
\text { Suryana, A., } \\
\text { Mulyana, S., } \\
\text { Hidayat, M. }\end{array}$ & 2019 & $\begin{array}{l}\text { Library } \\
\text { Philosophy and } \\
\text { Practice }\end{array}$ & $\begin{array}{l}\text { Review } \\
\text { (discursive) }\end{array}$ & $\begin{array}{l}\text { public opinion, social } \\
\text { media monitoring, } \\
\text { industry } 4.0 .\end{array}$ \\
\hline $\begin{array}{l}\text { Siano, A., } \\
\text { Vollero, A., } \\
\text { Della Volpe, } \\
\text { M., Confetto, } \\
\text { M.G., Foroudi, } \\
\text { P., Palazzo, M. }\end{array}$ & 2018 & Bottom Line & $\begin{array}{l}\text { Review } \\
\text { (theoretical) }\end{array}$ & $\begin{array}{l}\text { information, critical } \\
\text { analysis, communication } \\
\text { mix, integrated corporate } \\
\text { communication, message } \\
\text { coordination, physical } \\
\text { metaphors. }\end{array}$ \\
\hline $\begin{array}{l}\text { Abratt, R., } \\
\text { Cullinan, J. }\end{array}$ & 2017 & $\begin{array}{l}\text { Emerald } \\
\text { Emerging } \\
\text { Markets Case } \\
\text { Studies }\end{array}$ & $\begin{array}{l}\text { Review (case } \\
\text { study) }\end{array}$ & $\begin{array}{l}\text { marketing, corporate } \\
\text { communications, brand } \\
\text { management/equity. }\end{array}$ \\
\hline
\end{tabular}

Note: Sorted by number of citations (from highest to lowest)

\subsection{Strategic communication during Coronavirus pandemic: Opportunities and challenges}

\subsubsection{Dialogic communication during the crisis}

The Coronavirus pandemic (Covid-19) has had an impact on political and socio-economic structures and on their communication processes (Xifra, 2020). It has affected the institutions and organizations' stakeholder engagement. As a result, their communications and public relations departments had to respond to an unprecedented Covid-19 situation. The information they received about health, safety, and continuity was not always consistent and trustworthy 
(WHO, 2020). Yet, they had to engage with stakeholders, including employees as well as customers, to restore their faith and trust, thereby providing a sense of stability to them (McKinsey, 2020). They were expected to communicate in a clear and straightforward manner and as frequently as possible about their preventative social and fiscal measures in order to restore confidence in their organization.

In many cases, governments, institutions, and organizations including businesses and media have provided useful information on social distancing and hygienic practices to the general public. They encouraged citizens to remain calm and stay safe. This was the moment of truth for them to engage in dialogic communications with their publics (Wang \& Yang, 2020; Yang, Kang \& Cha, 2015; Capriotti \& Kuklinski, 2012; Yang, Kang \& Johnson, 2010; Bortree \& Seltzer, 2009; Seltzer \& Mitrook, 2007; Taylor, Kent \& White, 2001; Kent \& Taylor, 1998). The governments had to communicate with organizations and their citizens to instill trust in their preventative measures. Very often, they were following the recommendations of local and international health authorities (WHO, 2020). This is in stark contrast to previous crises situations, where institutions or organizations were not always responding to their stakeholder messages in a timely manner (Camilleri, 2017b; Roshan, Warren \& Carr, 2016).

The organizations' openness and willingness to engage in dialogic communication with stakeholders is usually evidenced during times of crises (Yang, Kang \& Johnson, 2010; Coombs, 2007). Crises are a source of reputational threat as organizations as well as institutions will usually react in different ways. For example, during COVID-19 various businesses have informed their employees about operational changes and new working conditions, revised their policies on leave of absence, working times, time-off, overtime, et cetera. Moreover, they established baseline safety requirements and adjusted to the new normal (McKinsey, 2020). Employees were instructed to maintain safe physical distances from each other, to adhere to 
cleaning and sanitizing procedures, and to ensure that they stay away from public activity, if they had temperature.

Very often, the businesses' corporate communications were positively framed as they highlighted best practices (Cheung \& Thadani, 2012; O’Keefe \& Jensen, 2008). Such communications could have been repeated and reinforced, as repetitive messages can have a persuasive effect on the individuals' perceived statement credibility (Kock \& Zerback, 2013; Segars \& Kohut, 2001). Conversely, there may be indirect and negative effects if repetition is taken too far, as individuals may not trust the source of the message (Rotfeld, 2006). The information service providers' legitimacy is a positive attribute that should be factored in, as reputable businesses can enhance public approvals, reinforce credibility and trust during crises (Lin, Spence, Sellnow \& Lachlan, 2016; Coombs, 2007; Fombrun, 2005). However, Yang et al. (2010) posited that source credibility as well as the salience of certain crises have minimal effects on postcrises outcomes. They argued that when it comes to crisis communication, the effective use of invitational rhetoric in openness to dialogic communication, may result in greater concerns and sympathy toward stakeholders. Crisis situations and extreme events with high threats and uncertainties will inevitably create challenges to detect credible and trustworthy information (Lin et al., 2016; Edwards, Spence, Gentile, Edwards \& Edwards, 2013).

\subsubsection{The use of the digital media for crisis communications}

Organizations can enhance their dialogic communications and relationships with stakeholders if they use digital media tools, including corporate websites, emails, blogs, social media, et cetera, on a consistent basis (Eriksson \& Olsson, 2016; Ngai \& Singh, 2014; Caron, Hwang, Brummans \& Caronia, 2013; Kristina \& Payal, 2013; Rybalko \& Seltzer, 2010). Relevant literature review suggests that social media have provided an interactive platform that fostered 
dialogic relationships between organizations and their publics (Wang \& Yang, 2020; Capriotti \& Kuklinski, 2012). Organizations can post status updates through social media (Killian \& McManus, 2015; Freberg, 2012) or may reply to their stakeholders' messages in real time (Men, Tsai, Chen \& Ji 2018; Grafström \& Falkman, 2017; Ott \& Theunissen, 2015). Several businesses can share links to websites and images through social media and disseminate news, information about products, events, announcements, et cetera, to capture the attention of their subscribers (Harlow, Salaverría, Kilgo \& García-Perdomo, 2017; Rybalko \& Seltzer, 2010). The organizations that post regularly updates on Twitter are experiencing more retweets and likes from the part of their followers (Wang \& Yang, 2020; Caerols-Mateo et al., 2017). Therefore, institutions and organisations are encouraged to use this medium or other social media, to engage in two-way communications with stakeholders and other publics. If they do so, their social media followers would feel valued, recognized and cared for. They would probably reinforce their sense of belonging when they interact with them (Wang \& Zhou, 2015).

However, during crises, institutions and organizations are not always utilizing these technologies in an appropriate manner (Jones, 2005). Perhaps, they did not allocate scarce resources to build up crisis communication capacities on social media platforms, or were not perceiving the benefits of using these interactive platforms with their audiences (Eriksson \& Olsson, 2016; Helsloot \& Groenendaal, 2013; Kavanaugh, Fox, Scheetz, Yang, Tzy, Shoemaker, Natsev \& Xie, 2012). Alternatively, they were not replying to their stakeholders in a timely manner, or they were not showing their 'humanness' (Roshan, Warren \& Carr, 2016).

Organizations need to be humane and responsive in their stakeholder engagement through the digital media. They should ensure that they address their followers' queries and concerns, particularly during times of crises. They will inevitably have to share distressing news on the 
state of their organization or about changes in their policies and practices, that can have devastating effects on stakeholders and other publics. For example, during COVID-19, organizations were not always in a position to communicate with certainty on when they will lift their social distancing measures. However, they were expected to be as credible and transparent as possible in their communications in order to instil trust and restore confidence (Colbran, 2020). Their effective communication of crisis narratives can reduce negative emotions and may even enhance positive post-crisis outcomes (Yang et al., 2010; Coombs, 2007). In this light, institutions and organizations can utilize social media to disseminate online content, including images, videos, live streams, et cetera, to engage with stakeholders. For example, they can use them to thank their employees' commitment or express their gratitude to frontline employees who faced threats to their health and safety during COVID-19. Alternatively, they may interact with them in one-on-one conversations via social media or messengers, to boost their morale and sense of belonging. Such dialogical communications can impart positive attitudes, improve the organizational culture and enhance stakeholder relationships.

\subsection{Conclusions}

The latest advances in the digital technologies have created significant challenges to identify truthful and trustworthy information, especially during times of crises. Institutions and organizations ought to be credible in their dialogical communications if they want to reinforce their legitimacy in society. There is scope for them to use the digital media, including corporate websites, emails, blogs, social media, et cetera, to reach diverse audiences. The social media is an important tool for crisis communications as it enables organizations to share their latest verbal, vocal and visual content including videos and live streams. Hence, corporate 
communications departments should incorporate digital and social media into their crisis and risk management plans. Social media enable subscribers to disseminate content in a viral manner and/or to actively engage in online conversations with individuals and organizations in real time. This implies that organizations are expected to monitor misinformation or fake news and to interact with their social media followers.

Despite the promise that digital media improves the efficiency and effectiveness of corporate communication, the practitioners' engagement with these technologies is neither automatic nor easy. The dialogic features that are enabled by them may (or may not) always result in improved stakeholder relationships.

\section{References}

Abratt, R. \& Cullinan, J. (2017). 5FM: youth radio in the digital age, Emerald Emerging Markets Case Studies, 7(2), https://doi.org/10.1108/EEMCS-05-2016-0074

Bachmann, P. (2019). Public relations in liquid modernity: How big data and automation cause moral blindness, Public Relations Inquiry, 8(3), 319-331.

Beltrá, M.N., Medina, I.G. \& Correia, P P. (2020), The Dialogical Potential of Facebook: The Case of Fashion Brands, International Journal of Interactive Mobile Technologies, 14(4), 3043.

Bortree, D.S. \& Seltzer, T. (2009), Dialogic strategies and outcomes: An analysis of environmental advocacy groups' Facebook profiles, Public Relations Review, 35(3), 317-319

Brennan, N. M. \& Merkl-Davies, D. M. (2018), Do firms effectively communicate with financial stakeholders? A conceptual model of corporate communication in a capital market context, Accounting and Business Research, 48(5), 553-577.

Bruce, M. \& Solomon, M. R. (2013), Managing for media anarchy: A corporate marketing perspective, Journal of Marketing Theory and Practice, 21(3), 307-318.

Butler, A. Camilleri, M.A., Creed, A. \& Zutshi, A. (2020). The use of mobile learning technologies for corporate training and development: A contextual framework. In Camilleri, M.A. (Ed.), Strategic Corporate Communication in the Digital Age, Emerald, UK.

Caerols-Mateo, R. Viñarás-Abad, M. \& Gonzálvez-Valles, J.E. (2017), Social networking sites and museums: analysis of the Twitter campaigns for 
International Museum Day and Night of Museums, Revista Latina de Comunicación Social, 72), 220-234.

Camilleri, M. A. (2015). The business case for corporate social responsibility. In Marketing \& public policy as a force for social change conference (pp. 8-14), American Marketing Association Conference Proceedings, Washington D.C, USA.

Camilleri, M.A. (2016a). Corporate Social Responsibility Reporting in Europe. In Key Initiatives in Corporate Social Responsibility (pp. 21-41). Springer, Cham.

Camilleri, M.A. (2016b). Corporate sustainability and responsibility toward education. Journal of Global Responsibility, 7(1),56-71.

Camilleri, M. A. (2016c). Reconceiving corporate social responsibility for business and educational outcomes. Cogent Business \& Management, 3(1),

https://www.cogentoa.com/article/10.1080/23311975.2016.1142044

Camilleri, M. A. (2017a). Corporate citizenship and social responsibility policies in the United States of America. Sustainability Accounting, Management and Policy Journal, 8(1),77-93.

Camilleri, M. A. (2017b). Corporate Sustainability, Social Responsibility and Environmental Management. Springer, Heidelberg, https://link.springer.com/book/10.1007\%2F978-3-319$\underline{46849-5}$

Camilleri, M.A. (2018a), Theoretical insights on integrated reporting: The inclusion of nonfinancial capitals in corporate disclosures, Corporate Communications: An International Journal, 23 No. 4), 567-581.

Camilleri, M.A (2018b). Unlocking Corporate Social Responsibility Communication through Digital Media. In Lindgreen, A., Vanhamme, J., Maon, F. and Watkins, R. (Eds)

Communicating Corporate Social Responsibility in the Digital Era. Routledge Oxford, UK.

Camilleri, M.A. \& Costa, R.A. (2018), The Small Businesses' Responsible Entrepreneurship and their Stakeholder Engagement through Digital Media. In Costa, C. (Ed.) 13th European Conference on Innovation and Entrepreneurship (ECIE2018). University of Aveiro, Aveiro, Portugal.

Camilleri, M. A. (2019). The online users' perceptions toward electronic government services. Journal of Information, Communication and Ethics in Society, 18(2), 221-235.

Camilleri, M. A. (2019). Measuring the corporate managers' attitudes towards ISO's social responsibility standard. Total Quality Management \& Business Excellence, 30(13-14), 15491561.

Camilleri, M. A. (2020). The market for socially responsible investing: A review of the developments. Social Responsibility Journal.

https://www.emerald.com/insight/content/doi/10.1108/SRJ-06-2019-0194/full/html

Camilleri, M.A. \& Isaias, P. (2020). The businesses' interactive engagement through digital media. In Camilleri, M.A. (Ed.), Strategic Corporate Communication in the Digital Age, Emerald, UK.

Capriotti, P. \& Kuklinski, H.P. (2012), Assessing dialogic communication through the Internet in Spanish museums, Public Relations Review, 38(4), 619-626. 
Capriotti, P., Zeler, I. \& Camilleri, M.A. (2020). Corporate communication through social networks: The identification of key dimensions for dialogic communication. In Camilleri, M.A. (Ed.), Strategic Corporate Communication in the Digital Age, Emerald, UK.

Caron, A.H., Hwang, J.M., Brummans, B.H.J.M. \& Caronia, L. (2013), Business writing on the go: How executives manage impressions through e-mail communication in everyday work life, Corporate Communications: An International Journal, 18 No. 1), 8-25.

Champoux, V., Durgee, J. \& McGlynn, L. (2012), Corporate Facebook pages: when 'fans' attack, Journal of Business Strategy, 33(2), 22-30.

Cheung, C.M. \& Thadani, D.R. (2012), The impact of electronic word-of-mouth communication: A literature analysis and integrative model", Decision Support systems, 54(1), 461-470.

Chu, S.C. \& Kim, Y. (2011), Determinants of consumer engagement in electronic word-ofmouth (eWOM) in social networking sites, International journal of Advertising, 30(1), 47-75.

Colbran, M. P. (2018), Policing, social media and the new media landscape: can the police and the traditional media ever successfully bypass each other? Policing and Society, 30(3), 295-309.

Coombs, W.T. (2007), Protecting organization reputations during a crisis: The development and application of situational crisis communication theory, Corporate Reputation Review, 10(3), 163-176.

Cornelissen, J. P. (2008). Corporate communication. The International Encyclopedia of Communication. https://onlinelibrary.wiley.com/doi/abs/10.1002/9781405186407.wbiecc143

Costa-Sánchez, C. \& Míguez-González, M.I. (2018), Use of social media for health education and corporate communication of hospitals", El Profesional de la Información, 27(5), 1145 1154.

Edwards, C., Spence, P.R., Gentile, C.J., Edwards, A. \& Edwards, A. (2013), How much Klout do you have? A test of system generated cues on source credibility, Computers in Human Behavior, 29, pps. A12eA16.

Eriksson, M. \& Olsson, E.K. (2016), Facebook and Twitter in crisis communication: A comparative study of crisis communication professionals and citizens, Journal of Contingencies and Crisis Management, 24(4), 198-208.

Dyuzhev, V.G. \& Boichenko, O.I. (2019), Influence of corporate social networks on the innovative receptivity of personnel, by example of Bitrix24 software (VO OVEN Ltd), Science Innovation, 15(2), 55-62.

Eisenhardt, K. M., Graebner, M. E., \& Sonenshein, S. (2016). Grand challenges and inductive methods: Rigor without rigor mortis, 59(4), 1113-1123.

Fombrun, C.J. (2005), A world of reputation research, analysis and thinking-building corporate reputation through CSR initiatives: evolving standards, Corporate Reputation Review, 8(1), 7-12.

Fraustino, J.D. \& Connolly-Ahern, C. (2015), Corporate associations written on the wall: Publics' responses to Fortune 500 ability and social responsibility Facebook posts, Journal of Public Relations Research, 27(5), 452-474. 
Freberg, K. (2012), Intention to comply with crisis messages communicated via social media, Public Relations Review, 38(3), 416-421.

García García, M., Carrillo-Durán, M.V. \& Tato Jimenez, J.L. (2017), Online corporate communications: website usability and content, Journal of Communication Management, 21 No. 2), 140-154.

García-Orosa, B. (2019), 25 years of research in online organizational communication: Review article. El Profesional de la Información (EPI), 28(5), 1-19.

Gorge, M. (2006), Mobility and security: two sides of the same coin, Computer Fraud and Security, Vol 11), 15-18.

Grafström, M. \& Falkman, L.L. (2017), Everyday narratives: CEO rhetoric on Twitter, Journal of Organizational Change Management, 30 No. 3), 312-322.

Hajarian, M., Camilleri, M.A.. Diaz, P \& Aedo, I. (2020). A taxonomy of online marketing methods for corporate communication. In Camilleri, M.A. (Ed.), Strategic Corporate Communication in the Digital Age, Emerald, UK.

Harlow, S., Salaverría, R., Kilgo, D. K. \& García-Perdomo, V. (2017), Protest paradigm in multimedia: Social media sharing of coverage about the crime of Ayotzinapa, Mexico, Journal of Communication, 67), 328-349.

Helsloot, I. \& Groenendaal, J. (2013), Twitter: An Underutilized Potential During Sudden Crises?', Journal of Contingencies and Crisis Management, 21(3), 178- 183.

Hoffmann, C.P. \& Aeschlimann, L. (2017), Shielding or engaging: the use of online shareholder platforms in investor relations, Corporate Communications: An International Journal, 22 No. 1), 133-148.

Holliman, G. \& Rowley, J. (2014), Business to business digital content marketing: marketers' perceptions of best practice, Journal of Research in Interactive Marketing, 8 No. 4), 269-293.

Järvinen, J. \& Taiminen, H. (2016), Harnessing marketing automation for B2B content marketing, Industrial Marketing Management, 54), 164-175.

Jones, S. L. (2005), From writers to information coordinators: Technology and the changing face of collaboration, Journal of Business and Technical Communication, 19(4), 449-467.

Kavanaugh, A.L., Fox, E.A., Scheetz, S.D., Yang, S., Tzy Li, L., Shoemaker, D.J., Natsev, A. \& Xie, L. (2012), Social Media Use by the Government: From the Routine to the Critical, Government Information Quarterly, 29(4), 480- 491.

Kent, M. L. \& Taylor, M. (1998), Building dialogic relationships through the World Wide Web, Public Relations Review, 24(3), 321-334.

Killian, G. \& McManus, K. (2015), A marketing communications approach for the digital era: Managerial guidelines for social media integration, Business Horizons, 58(5), 539-549. 
Koch, T. \& Zerback, T. (2013), Helpful or harmful? How frequent repetition affects perceived statement credibility, Journal of Communication, 63(6), 993-1010.

Köhler, K. \& Zerfass, A. (2019), Communicating the corporate strategy: An international benchmark study in the UK, the USA, and Germany, Journal of Communication Management, 23 No. 4), 348-374.

Krishna, A. \& Vibber, K.S. (2017), Victims or conspirators? Understanding a hot-issue public's online reactions to a victim cluster crisis, Journal of Communication Management, 21 No. 3), 303-318.

Lestari, M. T., MSi, A. S., MIKom, S. M. \& Hidayat, M. (2019), Analysis of the use of Social Media Monitoring for the existence of public opinion (Case study on PT. Telkom Indonesia Tbk Digital Platform), Library Philosophy and Practice), 1-11.

Lin, X., Spence, P.R., Sellnow, T.L. \& Lachlan, K.A. (2016), Crisis communication, learning and responding: Best practices in social media, Computers in Human Behavior, 65), 601-605.

Loureiro, S.M.C. \& Gomes, D. G. (2016), Relationship between companies and the public on Facebook: The Portuguese and the Brazilian context, Journal of Promotion

Management, 22(5), 705-718.

Manfredi-Sánchez, J.L. (2019), Brand activism, Communication and Society, 32(4), 343359.

McKinsey (2020), A leader's guide: Communicating with teams, stakeholders, and communities during COVID-19, https://www.mckinsey.com/business-

functions/organization/our-insights/a-leaders-guide-communicating-with-teams-stakeholdersand-communities-during-covid-19\#

Melewar, T.C. \& Navalekar, A. (2002), Leveraging corporate identity in the digital age, Marketing Intelligence and Planning, 20 No. 2), 96-103.

Mendes-da-Silva, W., Christensen, T.E. \& Richardson, V. J. (2008), Determinants of internet financial disclosure in an emerging market: Lessons from Brazil, Corporate Ownership and Control, 5(2), 379-392.

Mohd-Sulaiman, A.N. \& Hingun, M. (2020), Liability risks in shareholders' engagement via electronic communication and social media, International Journal of Law and Management. In Press. https://doi.org/10.1108/IJLMA-06-2019-0137

Ngai, C.S.B. \& Singh, R.G. (2014), Communication with stakeholders through corporate web sites: An exploratory study on the CEO messages of major corporations in Greater China, Journal of Business and Technical Communication, 28(3), 352-394.

O'Keefe, D.J. \& Jensen, J.D. (2008), Do loss-framed persuasive messages engender greater message processing than do gain-framed messages? A meta-analytic review, Communication Studies, 59(1), 51-67. 
Ott, L. \& Theunissen, P. (2015), Reputations at risk: Engagement during social media crises, Public Relations Review, 41(1), 97-102.

Overton-de Klerk, N. \& Verwey, S. (2013), Towards an emerging paradigm of strategic communication: Core driving forces, Communicatio, 39(3), 362-382.

Rios Marques, I., Casais, B. \& Camilleri, M.A. (2020). The effect of macro celebrity and micro influencer endorsements on consumer-brand engagement on Instagram. In Camilleri, M.A. (Ed.), Strategic Corporate Communication in the Digital Age, Emerald, UK.

Romenti, S., Valentini, C., Murtarelli, G. \& Meggiorin, K. (2016), Measuring online dialogic conversations' quality: a scale development, Journal of Communication Management, 20 No. 4), 328-346.

Roshan, M., Warren, M. \& Carr, R. (2016), Understanding the use of social media by organizations for crisis communication, Computers in Human Behavior, 63), 350-361.

Rotfeld, H.J. (2006), Understanding advertising clutter and the real solution to declining audience attention to mass media commercial messages, Journal of Consumer Marketing, 23 No. 4), 180-181

Rowley, J. (2008), Understanding digital content marketing, Journal of Marketing Management, 24, Nos. 5-6), 517-540.

Ruehl, C.H. \& Ingenhoff, D. (2015), Communication management on social networking sites: Stakeholder motives and usage types of corporate Facebook, Twitter and YouTube pages, Journal of Communication Management, 19 No. 3), 288-302

Rybalko, S. \& Seltzer, T. (2010), Dialogic communication in 140 characters or less: How Fortune 500 companies engage stakeholders using Twitter, Public Relations Review, 36(4), 336-341.

Sedeke, K. \& Arora, P. (2013). "Top ranking fashion blogs and their role in the current fashion industry, First Monday (Chicago)), 1-32.

See, Z.S., Sunar, M.S., Ab Aziz, K. \& Kusnayat, A. (2018), Interactive panorama VR360 for corporate communications: An industrial scenario case study, International Journal of Integrated Engineering, 10. No. 6), 169-177.

SEJ (2020). The 7 Biggest Social Media Sites in 2020. Search Engine Journal. https://www.searchenginejournal.com/social-media/biggest-social-media-sites/\#close

Segars, A.H. \& Kohut, G.F. (2001), Strategic communication through the World Wide Web: An empirical model of effectiveness in the CEO's letter to shareholders. Journal of Management Studies, 38(4), 535-556.

Seltzer, T. \& Mitrook, M.A. (2007), The dialogic potential of weblogs in relationship building, Public Relations Review, 33(2), 227-229. 
Siano, A., Vollero, A., Della Volpe, M., Confetto, M.G., Foroudi, P. and Palazzo, M. (2018), The role of physical metaphors for decision-making in integrated corporate communication, The Bottom Line, 31 No. 1), 42-55.

Taylor, M., Kent, M.L. \& White, W.J. (2001), How activist organizations are using the Internet to build relationships, Public Relations Review, 27(3), 263-284.

Troise, C. \& Camilleri, M.A. (2020). The use of the digital media for marketing, CSR communication and stakeholder engagement. In Camilleri, M.A. (Ed.), Strategic Corporate Communication in the Digital Age, Emerald, UK.

Turner, A., Wilkie, F. \& Rosen, N. (2004), Virtual but visible: developing a promotion strategy for an electronic library, New Library World, 105 No. 7/8), 262-268.

Vokic, N. P. \& Vidovic, M. (2015), Managing internal digital publics: What matters is digital age not digital nativity, Public Relations Review, 41(2), 232-241.

Wang, Y. \& Yang, Y. (2020), Dialogic communication on social media: How organizations use Twitter to build dialogic relationships with their publics, Computers in Human Behavior, 104, In Press https://doi.org/10.1016/j.chb.2019.106183

Wang, Y. \& Zhou, S. (2015), How do sports organizations use social media to build relationships? A content analysis of NBA clubs' twitter use, International Journal of Sport Communication, 8), 133-148

Weder, F., Einwiller, S. \& Eberwein, T. (2019), Heading for new shores: impact orientation of CSR communication and the need for communicative responsibility, Corporate Communications, 24(2), 198-211.

WHO (2020), Getting your workplace ready for COVID-19, https://www.who.int/docs/default-source/coronaviruse/getting-workplace-ready-for-covid19.pdf?ua=1

Xifra, J. (2020), Corporate communication, public relations and reputational risk management in the days of Covid-19, Profesional de la Informacion, Vol 29(2 http://eprints.rclis.org/39942/

Yang, S.U., Kang, M. \& Johnson, P. (2010), Effects of narratives, openness to dialogic communication, and credibility on engagement in crisis communication through organizational blogs, Communication Research, 37(4), 473-497.

Yang, S.U., Kang, M. \& Cha, H. (2015), A study on dialogic communication, trust, and distrust: Testing a scale for measuring organization-public dialogic communication (OPDC), Journal of Public Relations Research, 27(2), 175-192.

Ye, Q., Law, R., Gu, B. \& Chen, W. (2011), The influence of user-generated content on traveler behavior: An empirical investigation on the effects of e-word-of-mouth to hotel online bookings', Computers in Human behavior, 27(2), 634-639. 
